\title{
A Wide Beam And Broad Band Microstrip Helical Antenna Array For Mobile Communication
}

\author{
Xiaofeng Xiong \\ Microwave and Millimeter Wave Engineering Research \\ Center, University of Science and Technology of China \\ Hefei, China \\ jsgyxxf@mail.ustc.edu.cn
}

\author{
Lingyun Zhou ,Weidong Chen \\ Microwave and Millimeter Wave Engineering Research \\ Center, University of Science and Technology of China \\ Hefei, China \\ lyzhou@ustc.edu.cn,wdchen@ustc.edu.cn
}

\begin{abstract}
A novel broadband microstrip helical antenna array with $1 * 8$ elements is designed based on traditional helical antenna. The helix is realized by connecting stacked multilayer microstrip circular arcs with via holes. Meanwhile, grounded coplanar waveguide(GCPW) to stripline is utilized for probe station measurement. The simulated impedance and radiation pattern are studied. The proposed antenna shows a wide impedance bandwidth from $9.8 t 012 \mathrm{GHz}$ for $\left|S_{11}\right|<-10 \mathrm{~dB}$, with wide $3 \mathrm{~dB}$ beamwidth of E-plane about $120^{\circ}$, respectively.
\end{abstract}

Keywords-helical antenna array;wide beam; broadband;

\section{INTRODUCTION}

Microstrip antenna has been widely used due to its small size, lightweight, low profile, low cost and easy conform, especially suited for using as small mobile terminal's antenna of mobile satellite communication and missile or rocket antenna. Many applications, such as, Chinese biosatellite Positioning system, GPS, telemeter and telecontrol system, all require an antenna that can provide uniform response over approximately the entire upper hemisphere and high gain at low-angle[1]-[2]. Because the beams of conventional microstrip antenna are narrow, for example, the typical 3- $\mathrm{dB}$ beamwidth and bandwidth of microstrip antennas are $70^{\circ}$ $\sim 100^{\circ}$ and $3 \%$, respectively[3]. These characteristics make it difficult for microstrip antennas to afford so many functions. For a long time, helical antennas are widely used as CP antennas because of their good circular polarization and wide bandwidth based on axial-mode[4]. A novel broadband circularly polarized microstrip helical antenna [5] can achieve good radiation performance by connecting stacked multilayer with vertical via holes. The normal mode helical antenna (NMHA) is a common antenna used on transceivers for wireless communications due to its omnidirectional radiation pattern, while its bandwidth is small [6]. In order to increase the bandwidth of the NMHA, we can set the working mode between the axial-mode and normal-mode.

In this paper, We propsed a X-band multilayer helical antenna array. The proposed antenna element is composed of three 3/4-turn open loops at various layers connected by via holes to form an axial-normal-mode helical structure to generate traveling wave radiation. The designed antenna is fed by a stripline to separate the radiating element from the feeding structure by a ground plane. To keep the beamwidth of E-plane a $1 * 8$ helical antenna array is built and demonstrated. This type of helical antenna array is convenient for integration applications. Simulation results show that this microstrip helical antenna has wide impedance bandwidth and wide E-plane beamwidth with cmpact size.

\section{ANTENNA DESIGN}

\section{A Single Element}

The configuration of the single microstrip antenna is show in Fig.1 with its parameters. The horizontal microstrip circular arc is chosen to be three quarters of an annular ring, so the thickness of the dielectric h equals to $S * 3 / 4$. From the traditional method, the parameters R(diameter) and S(pitch) can be obtained. $\mathrm{W}$ is the width of patch and can be initially assigned as $50 \mathrm{ohm}$ microstrip line width. The helical antenna is fed by a stripline to suppress the radiation from the feeding line. The initial values of antenna parameters can be obtained by a series of calculation, and then HFSS can be used to optimize the parameters to obtain a better radiation performance.

\section{B Antenna array}

To keep the beamwidth of the E-plane, we propose a $1 * 8$ array as shown in Fig.2, which is composed of 16 elements. A UWB feeding network and grounded coplanar waveguide (GCPW) to stripline transition for easy probe station testing. Distance between elements is $21 \mathrm{~mm}$ and the size of this array with the CPW test pads is $51 \times 184 \times 5.61 \mathrm{~mm}^{3}$.

Fig.2 shows the geometry of the antenna array. The array is composed by antenna elements and feeding network. The feeding network is supposed to be a power splitter which divides the input power and feed them into the antenna elements[7], it is desired to be frequency independent in the operating frequency band. However, the discontinuity of the feeding network, such as the $\mathrm{T}$ junction branchs, may cause reflection, the reflection can be reduced only if impedance matching is arrived at the branches[8]. So the characteristic impedance should be transformed before it is connected to the next grade of the feeding network. The widths of the striplines are tapered linearly from wide to narrow and the characteristic impedances vary from $50 \Omega$ to $100 \Omega$ gradually. The model is simulated by HFSS. Fig.3 illustrate simulated return loss of the feeding network by cutting the antenna but with $50 \Omega$ impedance load. The result show that the array can cover the most of the UWB band with return loss less than $10 \mathrm{~dB}$. The surface current distribution of the feeding network at $7 \mathrm{GHz}$ and $12 \mathrm{GHz}$ is demonstrated in Fig.4. The 
amplitudes of the currents of the feeding line connected to the second and the third element are less than those of the first element. This is mainly caused by the mutual coupling between adjacent microstip lines.
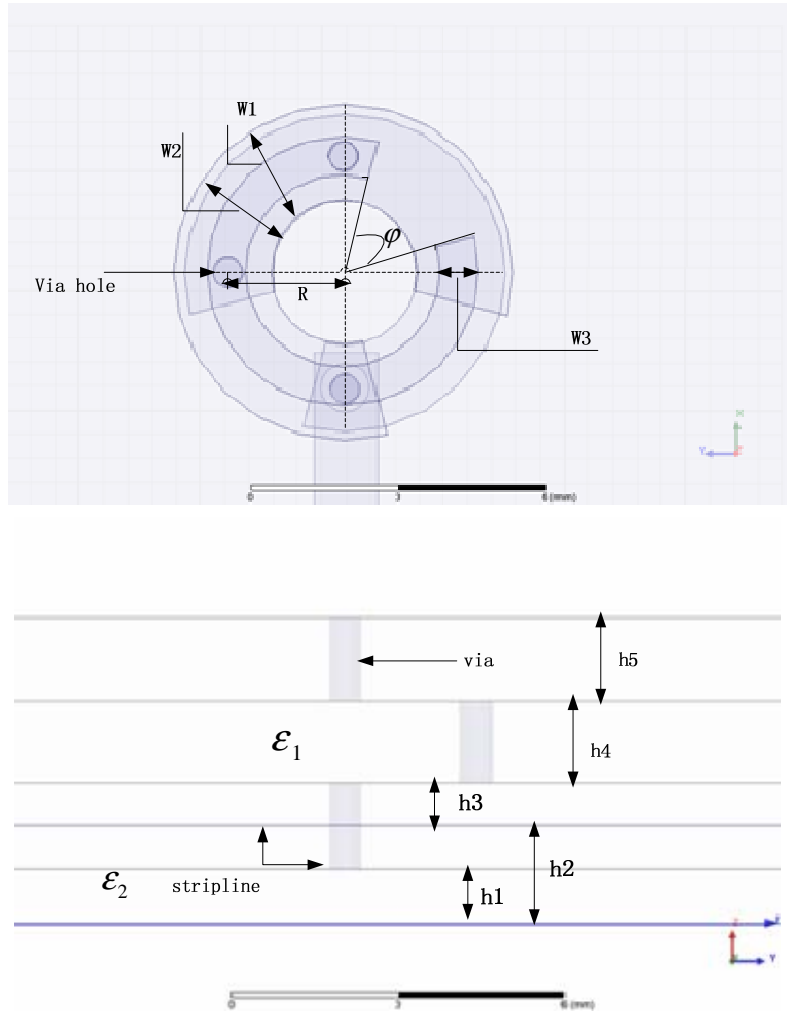

Fig.1 The configuration of the array element

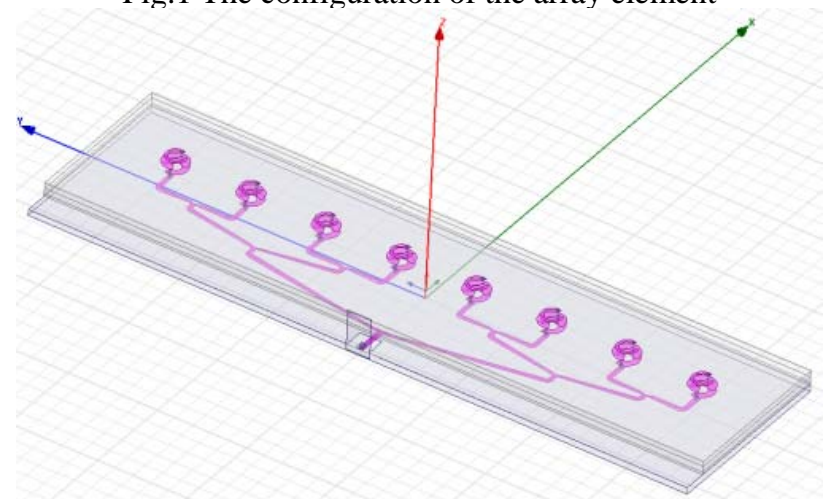

Fig.2 The Geometry of a $1 * 8$ helical antenna array.

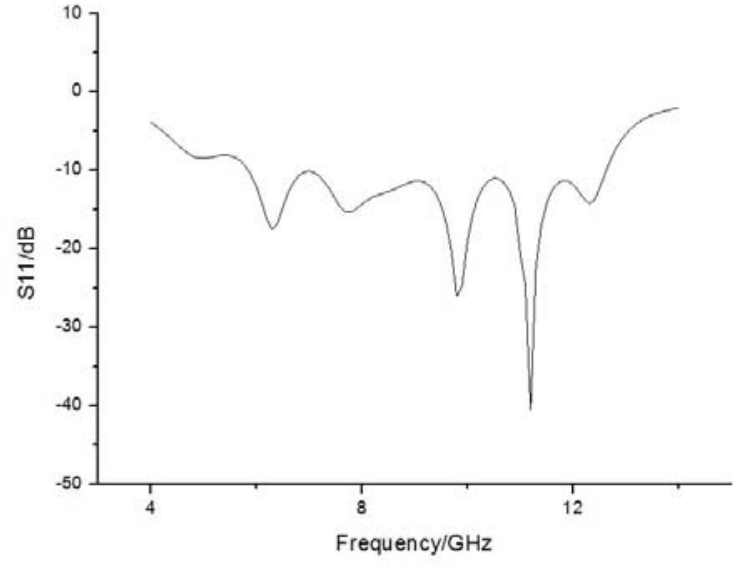

Fig.3 The return loss of the feeding network

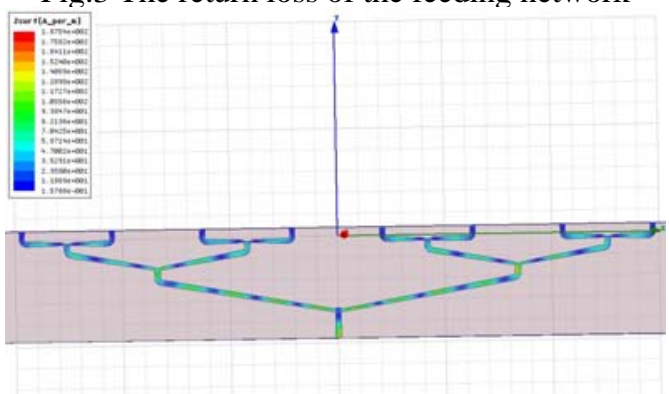

(a) $7 \mathrm{GHz}$

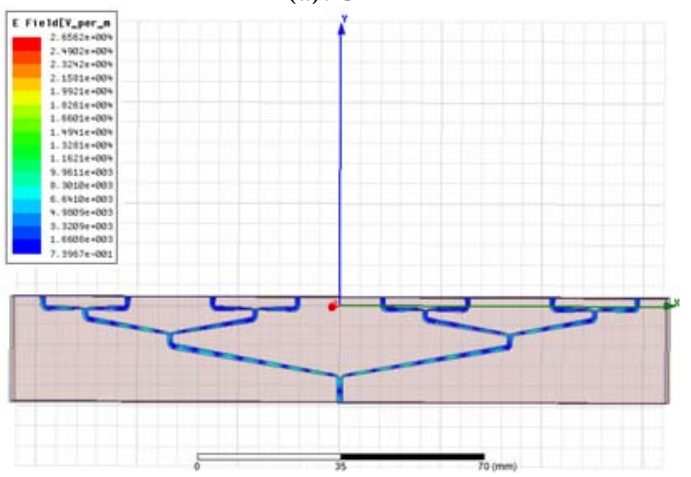

(b) $12 \mathrm{GHz}$

Fig.4 Surface current distribution of the array

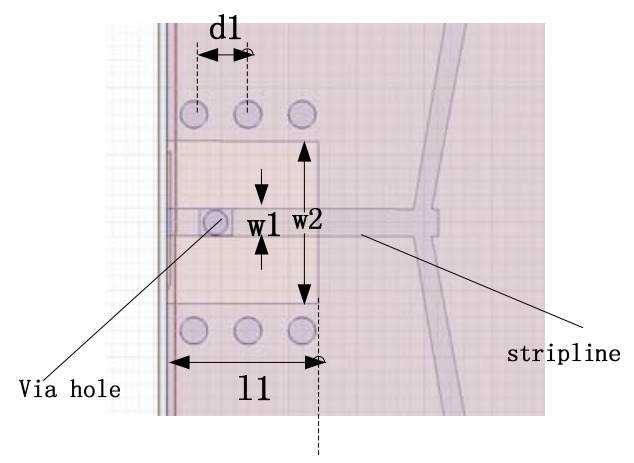




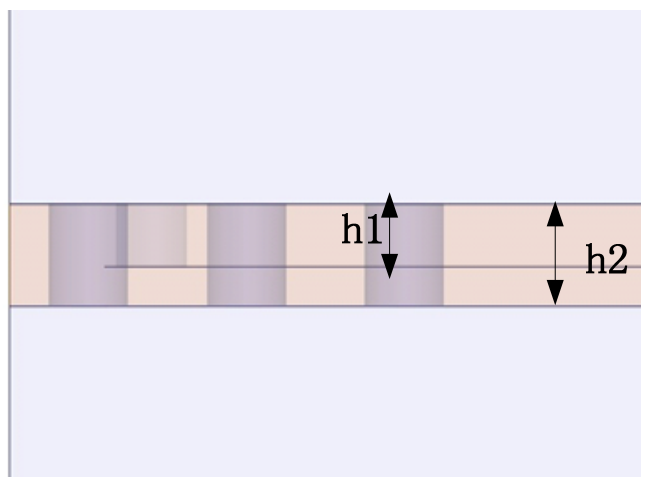

Fig.5 Geometry of GCPW to stripline transition. $\mathrm{d} 1=2 \mathrm{~mm}, \mathrm{l1}=5.6 \mathrm{~mm}, \mathrm{w} 1=1 \mathrm{~mm}, \mathrm{w} 2=6 \mathrm{~mm}, \mathrm{~h} 1=0.8 \mathrm{~mm}, \mathrm{~h} 2=1.3$ $\mathrm{mm}$

The CPW to stripline transition is illustrated in Fig.5, which is used for probe testing .

\section{RESULTS AND DISCUSSIONS}

Fig.6 shows the simulated return loss of the antenna array. It is seen that the simulated $|\mathrm{S} 11|$ is less than $-10 \mathrm{~dB}$ in the frequency range from $9.8 \mathrm{GHz}$ to $12.5 \mathrm{GHz}$, which is about $25 \%$ of the center frequency.

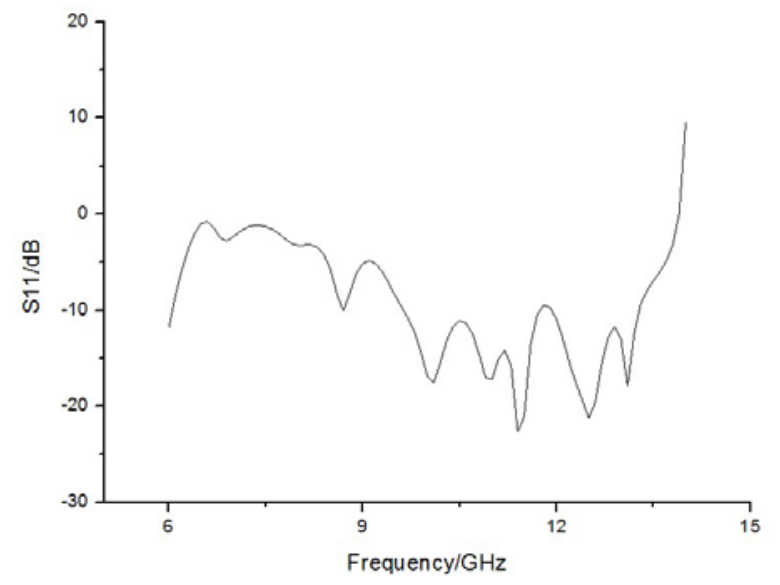

Fig.6 Simulated $|\mathrm{S} 11|$ of the antenna array

The radiation patterns of the antenna array is shown in Fig.7-9 at frequencies of $9.8 \mathrm{GHz}, 11 \mathrm{GHz}, 12 \mathrm{GHz}$,respectively, at the E-plane and H-plane. It can be seen that radiation patterns keep good directivity in wide bandwidth and shapes slightly. The E-plane keeps broad beamwidth in the array, While H-plane shapes narrow due to the application of the array.

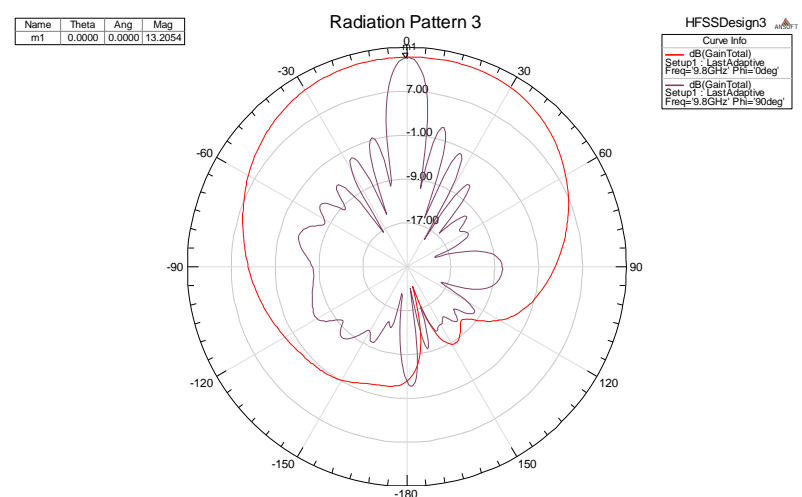

Fig.7 Radiation pattern of the antenna array at $9.8 \mathrm{GHz}$

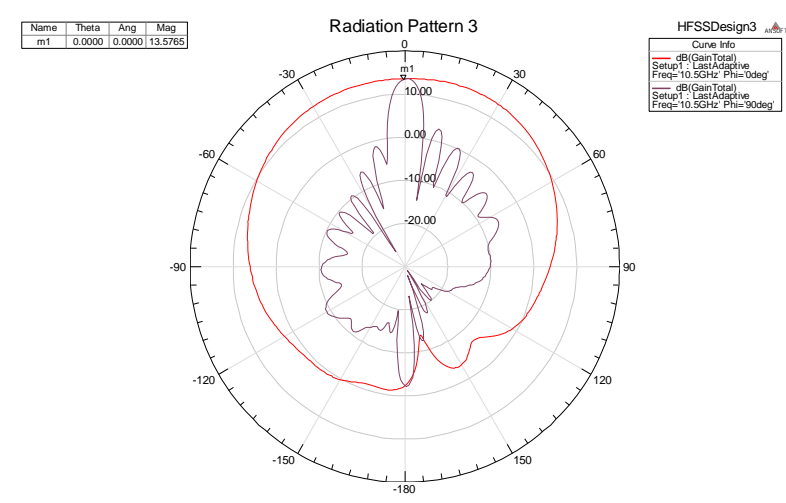

Fig.8 Radiation pattern of the antenna array at $11 \mathrm{GHz}$

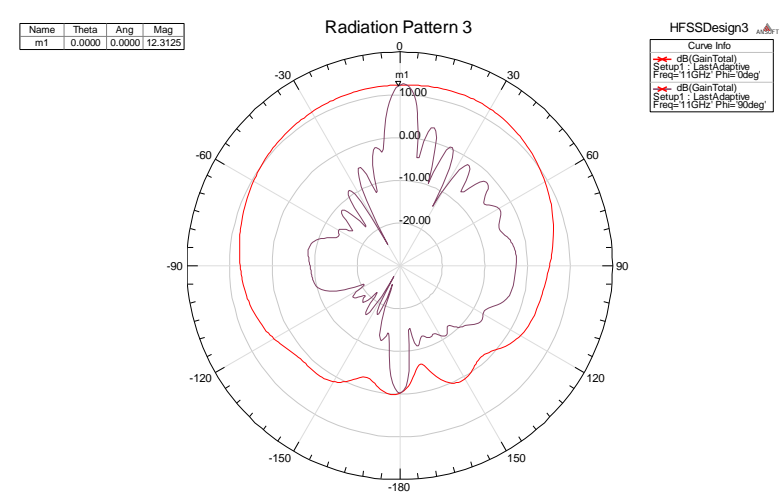

Fig.9 Radiation pattern of the antenna array at $12.5 \mathrm{GHz}$

Fig.10 shows the simulated gain of the antenna array.The gain of the array is between $11 \mathrm{dBi}$ to $13.5 \mathrm{dBi}$. The gain is improved compared with the antenna element. However, as the coupling effect and the loss of the feeding network increase with the increase of the frequency, the gain of the array decreases in the high frequency. 


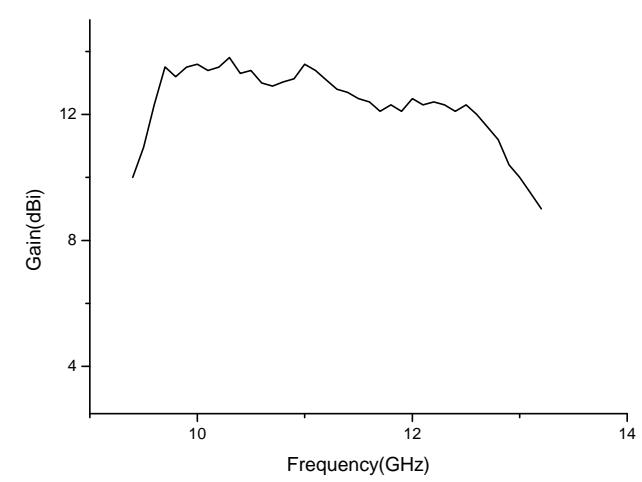

Fig.10 Gain of the $1 * 8$ antenna array

\section{CONCLUSION}

A 8-element microstrip helical antenna array is presented. The antenna takes advantage of traditional helical antennas and microstrip antennas. This antenna array has a wide bandwidth and broad beamwidth. The feeding network tapered linearly in order to get a match impedance in a wide band. The gain of the array is enhanced compared with the antenna element, especially in E-plane, which is $11 \mathrm{dBi}$ to
$13.5 \mathrm{dBi}$. The increase of mutual coupling and surface wave loss lead to the decrease of the antenna at high frequency.

\section{REFERENCES}

[1] Z. Qi and B. Liang, "Design of microstrip antenna with broader band width and beam,” in Proc. IEEE Int. Conf. Antennas and Propagation, vol. 3A, Washington, DC, USA, Jul. 2005, pp. 617-620.

[2] Meie Chen, Junhong Wang, "Planar UWB antenna array fed by microstrip network,”Chinese Journal of Radio Science, 2008,23(5), pp.911-916.

[3] Mao Wen-hui, Zhu Qi, "Design of cylindrical conformal millimeterwave microstrip antennas with broad beamwidth," Chinese Journal of Radio Science, vol. 23, no.2, April, 2008.

[4] Changrong Liu, Yong-Xin Guo, Xiaoyue Bao, Shao-Qiu Xiao, "60GHz ltcc integrated circularly polarized helical antenna array,” IEEE Trans. Antennas Propag., vol. 60, no. 3, pp. 1329-1335, March. 2012.

[5] Y. Y. Du and F. L. Liu, "A novel broadband circularly polarized microstrip helical antenna,” in Proc. 8th Int. Symp. Antennas, Propag. EM Theory, 2008, pp. 374-376.

[6] S. D. Rogers, 1. C. Young, C. M. Butler "Bandwidth enhanced normal mode helical antennas", Digest 1998 USNC/NRSI National Radio Science Meeting, Atlanta, GA. p 293 June 1998.

[7] Meie Chen, Junhong Wang, "Planar UWB antenna array with CPW feeding network,” Proceedings of APMC2008, 2008, pp.1-4.

[8] Maksimovitch Ye.,"UWB antenna array development for gpr applicatins," International Conference on Antenna Theory and Techniques, September, 2007, Sevastopol, pp.348-350. 